\title{
Familial hemiplegic migraine in the west of Scotland: a clinical and genetic study of seven families
}

\author{
M A S Ahmed, Evan Reid, A Cooke, Reynir Arngrímsson, J L Tolmie, J B P Stephenson
}

Department of Paediatric Neurology and Child

Development, Royal Hospital for Sick Children, Yorkhill NHS Trust, Glasgow, UK

M A S Ahmed

J B P Stephenson

Duncan Guthrie Institute of Medical Genetics, Yorkhill NHS Trust, Glasgow, UK

E Reid

A Cooke

R Arngrimsson

J L Tolmie

Department of Medical Genetics, University of Cambridge, UK E Reid

The first two authors (M A S A and E R) contributed equally to this paper.

Correspondence to: Dr E Reid, Department of Medical Genetics, Box 134, Addenbrooke's NHS Trust, Cambridge CB2 2QQ UK

Received 2 July 1996 and in revised form 7 August 1996 Accepted 8 August 1996

\begin{abstract}
Objectives-Clinical and genetic characterisation of families in the west of Scotland with familial hemiplegic migraine.

Methods-Families with familial hemiplegic migraine were identified via probands attending the regional paediatric neurology and child development centre. All available family members were assessed clinically and genetic linkage studies for the known familial hemiplegic migraine gene locus on chromosome 19 were carried out on three families.

Results-Seven unrelated kindreds with familial hemiplegic migraine were identified. Clinical information was obtained on 138 family members, 27 of whom fulfilled the International Headache Society criteria for familial hemiplegic migraine. Whereas the severity, duration, frequency, and temporal progression of acute hemiplegic migrainous attacks showed pronounced variability within and between families, and even in the same individual over time, no true clinical heterogeneity of the condition was apparent. Genetic linkage analysis gave results consistent with linkage to the familial hemiplegic migraine gene locus on chromosome 19p in one family. In the other two families, evidence against linkage was obtained. There was no significant clinical difference between these three families.

Conclusions-This study provides characterisation of the clinical features of familial hemiplegic migraine in a British population. Significant variability was found in the frequency and character of migraine attacks within and between families, and no true clinical heterogeneity was identified. On the other hand, further evidence for genetic heterogeneity of the condition was found.
\end{abstract}

$(\mathcal{F}$ Neurol Neurosurg Psychiatry 1996;61:616-620)

Keywords: Familial hemiplegic migraine; genetics; chromosome $19 \mathrm{p}$

Familial hemiplegic migraine is considered by the International Headache Society (diagnostic code $1 \cdot 2 \cdot 3$ ) to be a rare subtype of migraine with aura, transmitted in an autosomal dominant fashion, in which hemiplegia is the princi- pal aura feature. ${ }^{1}$ Over 40 families affected by the condition have been described worldwide, ${ }^{2-8}$ although its exact frequency is not documented. Familial hemiplegic migraine is clinically heterogeneous, with some affected members in a proportion of families having permanent and progressive neurological features such as nystagmus, deafness, tremor, and ataxia..$^{9-13}$

Considerable attention has been focused on the disease because it provides clear evidence that genetic factors are important in at least some types of migraine. A gene for familial hemiplegic migraine has recently been assigned to a 12 centimorgan region at chromosome $19 \mathrm{p} 12,{ }^{13}$ the first "migraine gene" to be located. However, the condition is genetically heterogeneous, being linked to this region in only eight out of 14 published families. ${ }^{12-15}$ Four of these families contained members with interictal cerebellar signs and all four were of the linked type, but otherwise no clinical differences are apparent between families in which familial hemiplegic migraine is linked to chromosome $19 p$ and those in which it is not. ${ }^{12-16}$

So far, little clinical data and no genetic linkage data are available on familial hemiplegic migraine within the United Kingdom. We present clinical findings in seven families with the disease, along with genetic linkage results in three families.

\section{Subjects and methods}

Seven unrelated families with at least two members with hemiplegic migraine were identified via probands attending the regional paediatric neurology and child development centre. All of the families originated from the west of Scotland, although some members were geographically scattered. Pedigree information was obtained from the probands' parents and other close relatives. All available family members were interviewed, usually by two doctors (MASA and ER). In rare cases, when direct interview was not feasible, a history was taken by telephone. If relatives were dead or unavailable, information about their disease status was obtained from at least two other family members. Based on these interviews, family members were classified into migraineurs or non-migraineurs, and the migraineurs were then subclassified, according to International Headache Society criteria, into those with familial hemiplegic migraine, migraine with aura (previously classic migraine), and migraine without aura (previ- 
Pedigree diagrams for families $1-3$, in which linkage studies were carried out.

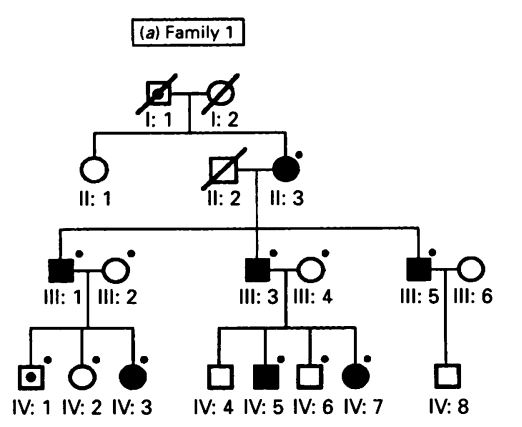

(b) Family 2
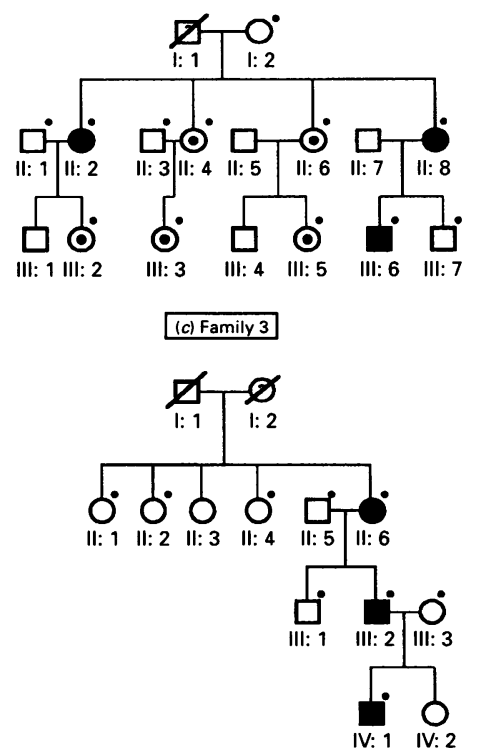

Familial hemiplegic migraine

Migraine with aura

? ? Possibly affected, on basis of history from other family members

- DNA sample available

ously common migraine). Hemiparesis was established by a history of inability to perform a motor function such as grip, writing, holding a cup, or walking. Lateralised dysfunction attributable to hemisensory disturbance was not regarded as hemiparesis. In members with familial hemiplegic migraine, data were gathered on age at onset, age at diagnosis, frequency and duration of attacks, side of weakness, aura symptoms, the temporal sequence of events, characteristics of the headache, time since the most recent attack, and trigger factors. All living patients with familial hemiplegic migraine were neurologically examined.

Three of the families (figure) were selected for genetic linkage analysis, because of their size and availability of samples. For these families, blood samples were collected from all available consenting family members. Genomic DNA was extracted by standard techniques. The polymerase chain reaction based dinucleotide repeat marker D19S226, located within the region of the familial hemiplegic migraine gene on chromosome $19 \mathrm{p}$, was used to genotype the families, as previously described. ${ }^{13}$ Two point LOD scores were calculated for each family using the LINKAGE computer programme. ${ }^{17}$ The mean age at onset for our patients with familial hemiplegic migraine was 14.7 years, with most affected by the age of 16 years, and so for linkage purposes clinically unaffected members under the age of 16 were classified as "unknown", as were those who had migraine with or without aura. Those who experienced attacks of both hemiplegic migraine and migraine with aura unaccompanied by weakness were classified as affected by familial hemiplegic migraine. Penetrance was assumed to be $90 \%$, in accordance with previously published results. ${ }^{13} \mathrm{~A}$ homogeneity test was performed with the HOMOG programme ${ }^{18}$ using linkage data from our families and from those previously published. ${ }^{13-15}$

\section{Results}

CLINICAL FINDINGS

Information was obtained on 138 family members in total. Twenty seven (13 male: 14 female) were identified as having familial hemiplegic migraine. Five of these had died before the study began and no clinical information is presented on them. A further six family members had migraine with aura, and nine had migraine without aura. Sixty eight family members were classified as unaffected and a further 28 were classified as having "unknown status", because they were clinically unaffected but younger than 16 .

The table summarises the clinical findings in the affected members. The mean age at onset of the condition was 13.6 (range 4-30) years, and the mean age at diagnosis was 31 (range 8-75) years. The frequency and severity of attacks was very variable within and between families. One family member, for example, had only ever experienced two attacks, whereas others had multiple attacks ranging in frequency from weekly to yearly. The duration of attacks ranged from two hours to seven days. By definition all patients experienced hemiplegia and it was exclusively right sided in six and exclusively left sided in 15. One patient had alternating weakness. For each member, the initial aura symptom tended to be consistent; in 15 patients it was a somatosensory symptom, such as numbness, heaviness, or tingling, whereas in the remaining seven it was a visual disturbance, such as teichopsia, coloured circles, fortification spectra, or scotomata. All patients experienced paraesthesia at some point during attacks, although its extent was variable. In 12 patients paraesthesia affected initially the hand and forearm, followed by the ipsilateral leg, foot, face, lips, and tongue. In three patients this sensory aura was restricted to the upper limb and ipsilateral face and in a further five it was restricted only to the limbs. Two patients were unsure of the exact site of paraesthesia. None of our patients experienced paraesthesia of the scalp, trunk, abdominal wall, or external genitalia. Dysarthria was experienced as an aura symptom in 20 patients. In 12 of these it was secondary to lingual paraesthesia, but in the remainder it was due to motor involvement. All patients had headaches of a throbbing 
Summary of clinical findings for patients with familial hemiplegic migraine

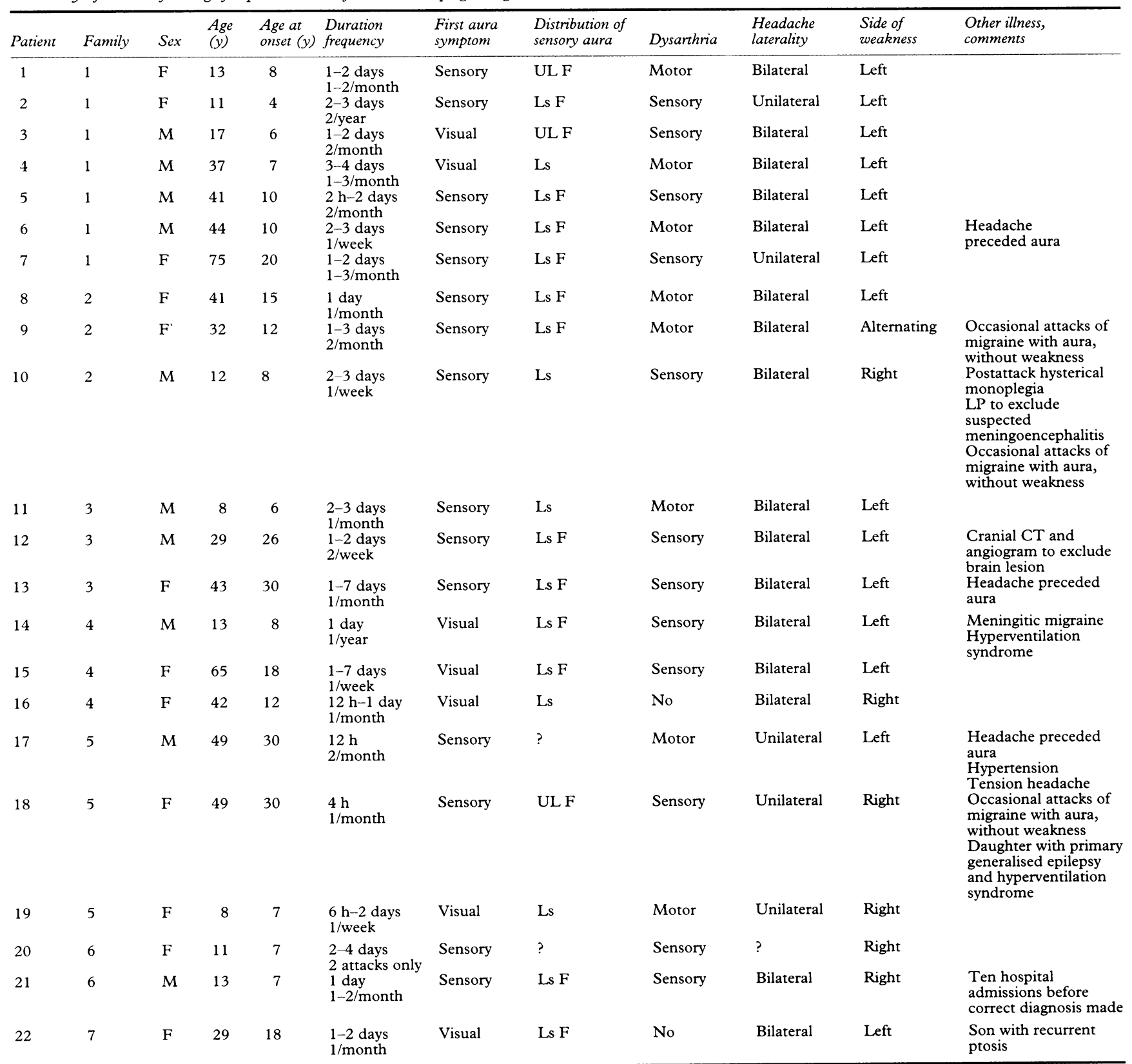

$\mathrm{UL}=$ Upper limb; Ls = ipsilateral upper and lower limbs; $\mathrm{F}=$ face.

quality, and these were bifrontal in 16 patients and unilateral in five One patient was unsure of headache laterality. In three patients the headache started before the aura and was shortly followed by neurological symptoms and signs. In one patient (10) an acute attack was accompanied by fever and coma lasting several days, necessitating inpatient admission to an intensive care unit.

Trigger factors were noted by 13 patients and the most common triggers were emotional upset and physical stress. Eating chocolate first thing in the morning was the main trigger in two patients (a father and daughter). In females, an increase in attack frequency was commonly attributed to pregnancy, commencement of the contraceptive pill, menstruation, and the menopause.

Interictal neurological examination was normal in all of the patients with familial hemi- plegic migraine, and in particular, no patient had cerebellar signs.

\section{GENETIC ANALYSIS}

A vertical pattern of inheritance consistent with autosomal dominant transmission was apparent in all of the families except one, in which two siblings with clinically normal parents were affected. In one family, both parents (17 and 18) of an affected child (19) gave a history of hemiplegic migraine, raising the possibility that she may have inherited two abnormal hemiplegic migraine genes, either at the same or different loci. Her clinical picture was not unusual. One symptomless obligate carrier was found. Hence, the penetrance in these families is estimated to be $96 \%(27 / 28)$, similar to previous reports in this condition.

A total of 33 members from families 1, 2, and 3 were genotyped using marker D19S226. 
In family 1 the maximum two point LOD score was 0.00 at recombination fraction (q) $0 \cdot 50$. Strong evidence against linkage was found in at least a $10 \mathrm{cM}$ region around this marker with a LOD score of -2.30 at $\theta=$ $0 \cdot 10$. Homogeneity testing using the HOMOG computer programme and results from previous publications showed that there was less than a $1 \%$ probability that this family was of the linked type. In family 2 strong evidence against linkage was also found with a LOD score of -3.03 at $\theta=0.0$ and a maximum LOD score of 0.00 at $\mathrm{q} 0.30$ to 0.50 . Evidence supporting linkage was only seen in family 3 , with a maximum LOD score of 0.82 at $\theta=0 \cdot 0$, with homogeneity testing giving an $87 \%$ chance of it being of the linked type.

\section{Discussion}

Familial hemiplegic migraine is defined as a subtype of migraine with aura in which affected members have hemiparesis as an aura symptom and in which at least one first degree relative has had similar attacks. ${ }^{1}$ It is commonly regarded as a rare condition, and only about 40 pedigrees have been published previously. ${ }^{2-8}$ However, we have identified 27 members from seven families originating in the west of Scotland who strictly fulfil the International Headache Society diagnostic criteria for familial hemiplegic migraine. All of the families were identified after referral of an affected child to the regional paediatric neurology referral centre, and so our study may represent an underestimate of the total number of families affected by the condition in the west of Scotland. Underrecognition of familial hemiplegic migraine is also suggested by the delay in diagnosis experienced by many patients. In our families, the average age of onset was about 14 years and the average age at diagnosis was 17 years later. In one person the correct diagnosis was made as late as 75 years of age (55 years after onset!), only after referral of an affected grandchild. Incorrect previous diagnoses in affected patients included cerebral vascular malformation, cerebral infarction, brain tumour, and meningitis.

In our patients with familial hemiplegic migraine, acute attacks were typical of a migraine with aura, with aura symptoms, including hemiplegia, usually being followed by headache. Non-headache symptoms of migraine, such as vomiting, nausea, abnormal bowel activity, abdominal pains, and food intolerance were uncommon. Rarely, attacks were atypical, leading to diagnostic confusion. In one child an acute attack was accompanied by fever and coma, with an aseptic pleocytic CSF. This attack necessitated inpatient admission to an intensive care unit. Although meningitic migraine has been reported, ${ }^{719}$ this diagnosis was delayed because at the time of presentation the family history of hemiplegic migraine had not been recognised. The diagnosis of "atypical" familial hemiplegic migraine should be considered in those with an appropriate family history.

Triggering factors are often reported by patients with migraine and previous studies have highlighted minor head trauma and cerebral angiography as triggering factors for familial hemiplegic migraine..$^{7021}$ Just over half of our patients with familial hemiplegic migraine reported triggering factors, most commonly physical and emotional stress. Triggering factors commonly associated with the more frequent types of migraine, such as the consumption of drugs, particular foods or food additives, alcohol, and soft drinks, were rarely reported.

Linkage results for the familial hemiplegic migraine locus on chromosome $19 p$ were available for three of our families and they are consistent with the previously recognised genetic heterogeneity of the condition. ${ }^{12-15}$ In two families, evidence against linkage to the familial hemiplegic migraine region on chromosome $19 p$ was found, whereas evidence supporting linkage to this region was found in a third family. Despite this evidence of genetic heterogeneity, there was no true clinical heterogeneity in our families; although there was a great deal of variation in the severity and frequency of attacks between affected members within the same family and in different families, none of our families contained members with the clinical features occasionally associated with hemiplegic migraine, such as cerebellar ataxia and nystagmus. Thus no correlation between genetic locus and clinical phenotype was apparent. This is consistent with findings from other published families in which all affected members lacked cerebellar involvement. Linkage of familial hemiplegic migraine to the chromosome $19 p$ locus has been confirmed in some of these families, but excluded in others. ${ }^{13-15}$ It seems remarkable that mutations in different genes may result in such a homogenous clinical picture. However, similar situations are well recognised for other genetic conditions, such as tuberous sclerosis, ${ }^{22}$ adult polycystic kidney disease, ${ }^{23}$ and familial spastic paraplegia. ${ }^{24}$ It may be that the different gene products are involved in the same biochemical pathway or a parallel biochemical pathway, or that they are constituents of the same protein complex. On the other hand, all of the familial hemiplegic migraine families containing members with cerebellar involvement tested to date have been of the linked type, suggesting that this clinical feature may be correlated with the chromosome 19 p genetic locus. ${ }^{12-15}$

Interestingly, multilocus analysis has placed the familial hemiplegic migraine gene on chromosome $19 p$ close to the genes for two other neurological conditions with a paroxysmal nature-cerebral autosomal dominant arteriopathy with subcortical infarcts and leukoencephalopathy (CADASIL) and hereditary paroxysmal cerebellar ataxia (HPCA). ${ }^{2526}$ Recently, mutations in several different membrane ion channel genes have been shown to underlie some neurological conditions with fluctuating or episodic symptoms and signs, ${ }^{27}$ and this has led to the suggestion that all three conditions might be allelic and that abnormalities in an ion channel gene may be responsi- 
ble. However, whereas the likely candidate region for the familial hemiplegic migraine gene on chromosome $19 p$ overlaps both those of CADASIL and HPCA, a recent mapping study strongly suggests that the likely candidate regions for the genes involved in CADASIL and HPCA do not overlap and so these two conditions are unlikely to be allelic. ${ }^{28}$

In conclusion, our study provides further characterisation of the clinical features of familial hemiplegic migraine and suggests that it is underrecognised. Whereas significant variability was found in the frequency and character of migraine attacks within and between families, no true clinical heterogeneity was identified. On the other hand, further evidence for genetic heterogeneity of the condition was found.

We gratefully acknowledge the families involved in this study for their help in its completion and Dr Charles ffrenchfor their help in its completion and Dr Charles ffrenchthank Jean Hislop for preparing the illustration.

1 Headache Classification Committee of the International Headache Society. Classification and diagnostic criteria for headache disorders, cranial neuralgias and facial pain Cephalalgia Suppl 1988;8:19-20.

2 Clarke JM. On recurrent motor paralysis in migraine, with report of a family in which recurrent hemiplegia accompanied the attacks. $B M \mathcal{F} 1910 ; 1: 1534-8$.

3 Whitty CWM. Familial hemiplegic migraine. $f$ Neurol Neurosurg Psychiatry1953;16:172-7.

4 Bradshaw P, Parsons M. Hemiplegic migraine, a clinical study. $Q \mathcal{F}$ Med 1965;133:65-84.

5 Heyk $\mathrm{H}$. Varieties of hemiplegic migraine. Headache 1973; 12:135-42.

6 Jensen TS, De Fine Olivarius B, Kraft M, et al. Familial hemiplegic migraine-a reappraisal and long term followup study. Cephalalgia 1981;1:33-9.

7 O'Hare JA, Feely MJ, Callaghan N. Clinical aspects of familial hemiplegic migraine in two families. Ir Med $\mathfrak{f}$ 1981;74:291-5

8 Haan J, Terwindt GM, Bos PLJ, et al. Familial hemiplegic migraine in the Netherlands. Clin Neurol Neurosurg 1994;96:244-9.

9 Zifkin B, Andermann E, Andermann F, et al. An autosomal dominant syndrome of hemiplegic migraine, nystagmus and tremor. Ann Neurol 1980;8:329-32.

10 Ohta M, Araki S, Kuroiwa Y. Familial occurrence of migraine with a hemiplegic syndrome and cerebellar manifestations. Neurology 1967;17:813-7.

11 Codina A, Acarini PN, Miguel F. Migraine hèmiplègique associèe à un nystagmus. Rev Neurol 1971;124:526-30.

12 Elliot MA, Peroutka SJ, Welch S, et al. Familial hemiplegic migraine, nystagmus and cerebellar atrophy. Ann Neurol 1996;39:100-6.

13 Joutel A, Bousser M-G, Biousse V, et al. A gene for familia hemiplegic migraine maps to chromosome 19. Nature genet 1993;5:40-5.

14 Joutel A, Ducros A, Vahedi K, et al. Genetic heterogeneity of familial hemiplegic migraine. Am $\mathcal{F}$ Hum Genet 1994; 55:1166-72.

15 Ophoff RA, Eijk RV, Sandkuijl LA, et al. Genetic heterogeneity of familial hemiplegic migraine. Genomics $1994 ; 22: 21-6$

16 Terwindt GM, Ophoff RA, Haan J, et al. Familial hemiplegic migraine: a clinical comparison of families linked and unlinked to chromosome 19. Cephalalgia 1996;16: 153-5.

17 Lathrop GM, Lalouel JM, Joulier C, et al. Multilocus linkage analysis in humans: detections of linkage and estimation of recombination. Am $\mathcal{f}$ Hum Genet 1985;37:482-98.

$18 \mathrm{Ott} \mathrm{J}$. Analysis of human genetic linkage. Baltimore: The Johns Hopkins University Press, 1993.

19 Fitzimons RB, Wolfenden WH. Migraine coma. Meningitic migraine with cerebral oedema associated with a new form of autosomal dominant cerebellar ataxia. Brain 1985;108:555-77.

20 Blau JN, Whitty CWM. Familial hemiplegic migraine. Lancet 1955;ii: $1115-6$.

21 Glista G, Mellinger JF. Familial hemiplegic migraine. Mayo Clin Proc 1975;50:307-11.

22 Sampson JR, Harris PC. The molecular genetics of tuberous sclerosis. Hum Mol Genet 1994;3:1477-80.

23 Schneider MC. Advances in polycystic kidney disease. Molecular Medicine Today 1996;2:70-5.

24 Kobayashi H, Garcia CA, Alfonso G, et al. Molecula genetics of familial spastic paraplegia: a multitude of responsible genes. $\mathcal{F}$ Neurol Sci 1996;137:131-8.

25 Tournier-Lasserve E, Joutel A, Melki J, et al. Cerebra autosomal dominant arteriopathy with subcortical infarcts and leukoencephalopathy maps to chromosome 19p12. Nature Genet 1993;3:256-9.

26 Vahedi $K$, Joutel A, Van Bogaert P, et al. A gene for hereditary paroxysmal cerebellar ataxia maps to chromosome tary paroxysmal cerebellar ataxia

27 Griggs RC, Nutt JG. Episodic ataxias as channelopathies. Ann Neurol 1995;37:285-7.

28 Ducros A, Alamowitch S, Nibbio A, et al. Cerebral autosomal dominant arteriopathy with subcortical infarcts and leukoencephalopathy, genetic homogeneity, and mapping of the locus within a 2-cM interval. Am $\mathcal{f}$ Hum Gene 1995;58:171-81. 